\title{
Coexistence of myasthenia gravis and neuromyelitis optica spectrum disorder: case report in Colombia and literature review
}

\begin{abstract}
Myasthenia Gravis (MG) and Neuromyelitis Optica Spectrum Disorder (NMOSD) are antibody-mediated channelopathies caused by complex immunological mechanisms, which are thus associated with multiple autoimmune diseases. This paper describes the case of a Colombian male patient with a history of seropositive MG that required thymectomy. After four years, he had an Optic Neuritis event and 30 years later, due to suspected autoimmune comorbidities, further investigations were performed, confirming the coexistence of NMOSD with presence of Anti-Aquaporin 4 (AQP4) antibodies (Abs).

Several reports in the literature show that the coexistence of these two pathologies is more common than expected by chance. Few cases have been reported in Latin America, which makes this report meaningful. In contrast to our case, the literature shows a higher incidence among women, but, on the other hand, it is consistent with an onset with mild or moderate MG symptoms at the clinical presentation, most of which led to thymectomy, followed by manifestations of NMOSD. Timely diagnosis and the consequent therapeutic adjustment may have a significant impact on the disability of these patients. There is a need for more reports in order to extend the statistical information and to improve the characterization of these patients, primarily in our Latin American region.
\end{abstract}

Keywords: myasthenia gravis, neuromyelitis optica spectrum disorder, autoimmune diseases, antibody-mediated disease, case report
Volume 10 Issue 3 - 2020

\author{
Natalia Gomez,' Luis Alexander Granados, ${ }^{2}$ \\ Wilson Andres Polo, ${ }^{3}$ Vivian Ximena \\ Mosquera, ${ }^{4}$ Maritza Lorena Arroyo ${ }^{5}$ \\ 'Physician and researcher of NeuroNet group, Colombia \\ ${ }^{2}$ Neurologyst and researcher of NeuroNet group, Colombia \\ ${ }^{3}$ Neurosurgeon and researcher of NeuroNet group, Colombia \\ ${ }^{4}$ Family Physician and researcher of NeuroNet group, Colombia \\ ${ }^{5}$ Physician, M.S. in Neuroscience, researcher of NeuroNet \\ groupColombia
}

Correspondence: Luis Alexander Granados, Neurologyst, NeuroNet Manager, Calle I 55 \# 9- 45 Bogotá, Colombia, Tel+573I I 845 I297,Email alexgranados8@gmail.com

Received: May 25, 2020 | Published: June 12, 2020
Abbreviations: MG, myasthenia gravis; NMOSD, neuromyelitis optica spectrum disorder; CNS, central nervous system; $\mathrm{AQP} 4$, aquaporin 4; AChR, acetylcholine receptor; MUSK, musclespecific kinase; Abs, antibodies; MRI, magnetic resonance image

\section{Introduction}

MG is an antibody-mediated autoimmune disease involving different components of the postsynaptic membrane of the neuromuscular junction, such as muscle-specific kinase (MUSK) and the acetylcholine receptor (AChR), the latter being present in 70-80\% of the cases, with clinical manifestations of weakness and fluctuating muscle fatigue.'

Its incidence and prevalence ranges from 0.8 to 1 and from 15 to 25 cases per 100,000 individuals, respectively, with a bimodal presentation of 20 to 40 years with female predominance, and 60 to 80 years, balanced between men and women. ${ }^{1,2}$ Patients with MG are associated with other autoimmune diseases between 5-30\%, with predominance of thyroid diseases such as Graves' disease and exhibit a higher incidence of demyelinating diseases compared to the general population. ${ }^{3,4}$

Neuromyelitis Optica (NMO) is a demyelinating disease of the Central Nervous System (CNS), mainly involving the optic nerve and spinal cord, mediated by antibodies targeted against the most abundant water channel protein in astrocytes, Aquaporine-4 (AQP4). These anti-AQP4 Abs, also called NMO IgG Abs, are used as a specific test for Neuromyelitis Optica Spectrum Disorders (NMOSD), with a specificity of $99-100 \%$ and a sensitivity of $58-91 \%$, though detection rates vary among different laboratory tests, with patients within the NMOSD group being seronegative.,
The prevalence of NMOSD ranges around 3.9 to 10 with an incidence of 0.07 to 0.73 per 100,000 individuals respectively; the difference between women and men is 8-9:1 (1). Higher associations with autoimmune diseases have been reported in seropositive NMOSD patients in up to $25-50 \%$ of cases (4), including Systemic Lupus Erythematosus (SLE), Sjögren's syndrome (SS), autoimmune thyroid diseases, and MG (5).

In this paper the case of a patient with a history of seropositive $\mathrm{MG}$, coexistence of NMOSD and presence of anti-AQP4 Abs is reported. Additionally, the relevant literature is reviewed.

\section{Case report}

Male patient, born in Bogotá, Colombia, with no personal or family history of autoimmunity or any other clinically meaningful pathology. At 16 years of age, the patient presented with diplopia and fluctuating weakness in 4 limbs. Myasthenia Gravis is diagnosed by electrodiagnostic tests and positive anti-AChR Abs. Three years after diagnosis, he underwent thymectomy (no pathology report available). The patient exhibits adequate adherence and response to treatment with pyridostigmine, achieving recovery of prior performance status and physical activity.

At 20 years of age, the patient presents with an event of blurred vision and pain in the right eye, he is assessed in the emergency room where optic neuritis is diagnosed and managed with pulses of methylprednisolone. Thirty years later, he attended our Neurology service for a control visit with symptoms of MG controlled with pyridostigmine. The following positive findings on physical examination were collected: a 20/30 visual acuity score (left eye), seeing shadows (right eye), ipsilateral afferent pupillary defect, with normal Intraocular pressure measured by Ophthalmology and 
evidence of disc pallor in the fundus of the right eye. There were no alterations in other cranial nerves, no alterations in strength or reflexes, normal sensitivity, with no evidence of fatigability or gait impairment. Additionally, there is evidence of changes in skin and skin appendages due to alopecia and madarosis. This, in association with the visual deficit derived from the optic neuritis event, makes the presence of autoimmune comorbidities suspicious, so further investigations are undertaken (Table 1) and CNS MRI was performed without abnormal findings. Anti-AChR Abs and anti-AQP4 Abs are positive, thus confirming the coexistence of MG and NMOSD in this patient. Considering the above, it was decided to add azathioprine to medical management, which showed adequate response and so far, no new relapses have emerged.

Table I Serum paraclinical studies

\begin{tabular}{llll}
\hline Test & Result & Test & Result \\
\hline HIV ELISA Test & Negative & Anti-dsDNA Antibodies & Negative \\
Glucose & Normal & Anti-Sm Antibodies & Negative \\
Potassium & Normal & Anti-RNP Antibodies & Negative \\
VDRL & Nonreactive & Rheumatoid factor & Negative \\
TSH & Normal & Anti-TPO Antibodies & Negative \\
Vitmain BI2 levels & Normal & Anticardiolipin Antibodies & Negative \\
Folic Acid & Normal & Anti-Acetylcholine Receptor Antibodies & Positive \\
Complete Blood count & Normal & Creatinine & Negative \\
AQP4-IgG & Positive & PTH & Negative \\
Antiphospholipid Antibodies & Negative & Iron & Negative \\
Anti-nuclear Antibodies & Negative & Serum Protein & Negative \\
Anti-SSA & Negative & Peripheral Smear & Negative \\
Anti-SSB & Negative & Erythrocyte Sedimentation Rate & Negative \\
\hline
\end{tabular}

HIV, human immunodeficiency viruses; ELISA, enzyme-linked immuno sorbent assay; VDRL, venereal disease research laboratory; TSH, thyroid stimulating hormone; AQP4, Aquaporin 4; SSA, Anti-Sjögren's-syndrome-related antigen A; SSB, Anti-Sjögren's-syndrome-related antigen B; dsDNA double stranded deoxyribonucleic acid; Sm, Smith; RNP, ribo nucleo protein;TPO, thyroid per oxidase; PTH, Parathyroid hormone

\section{Literature review and discussion}

The first case of NMO associated with autoimmune diseases was a woman with SLE in 1976. Since then, there have been reports regarding various pathologies sharing such antibody-mediated pathogenesis, where MG has been described (Figure 1). ${ }^{5}$ Studies state that the number of cases of NMOSD and MG are 70 times higher than expected by chance. ${ }^{4}$

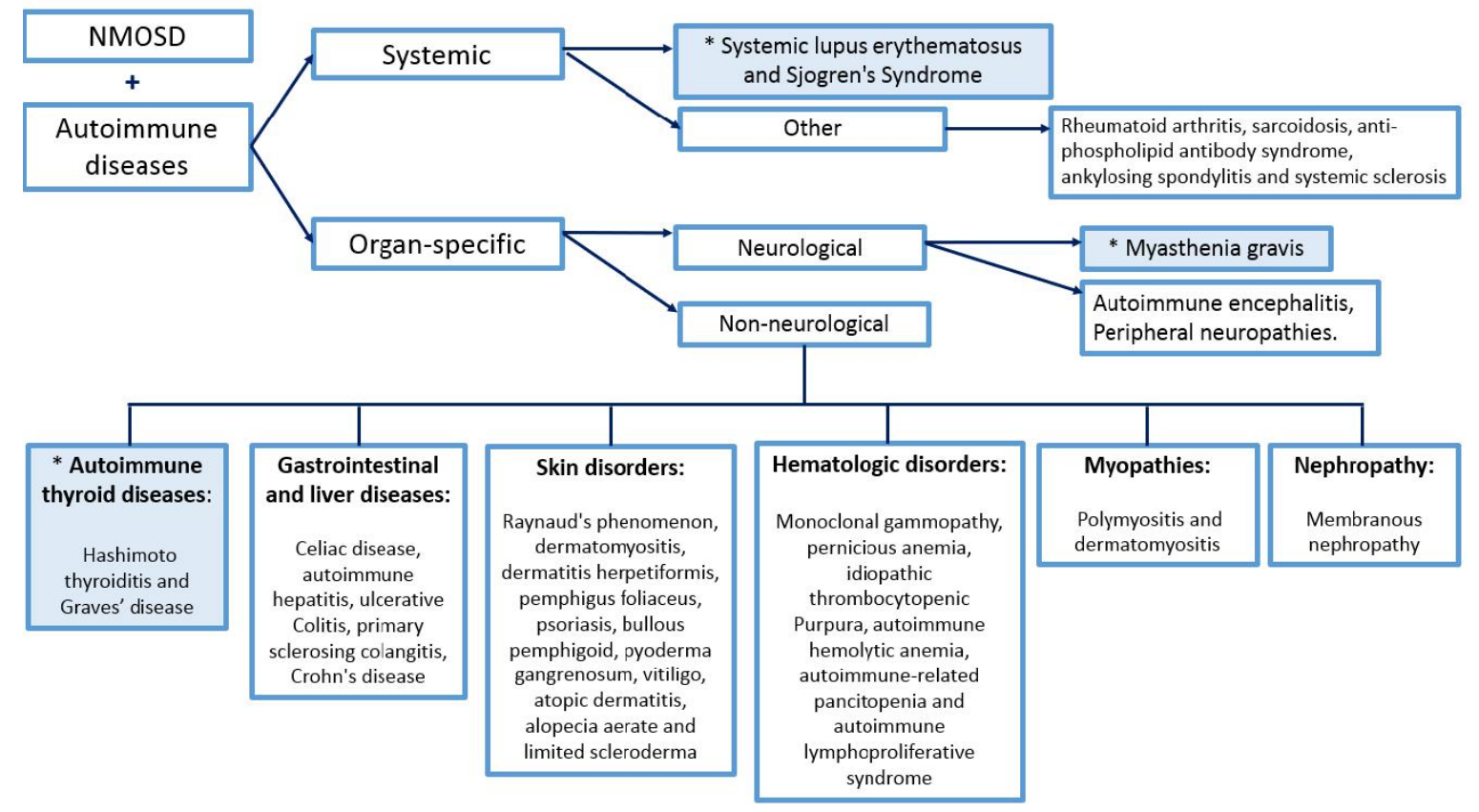

Figure I Coexistence of autoimmune diseases and NMOSD (* the most frequently reported diseases in each group). (Based on (5)). 
Both diseases are channelopathies caused by complex immunological mechanisms, mediated by the presence of Th1, Th17 and Thf lymphocytes. These end up promoting the production and maturation of B cells, which in turn produce self-reactive antibodies, causing complement activation and tissue damage. ${ }^{1}$

Most of the reports found in the literature describe Caucasian and Asian patients, with few reports in Latin America, mainly in Argentina and Brazil. Unlike the reported case, the literature describes female predominance. This has a biological rationale, apparently explained by inherent factors of gonadal hormones. Estrogens are believed to promote the presence of self-reactive B cells by acting on follicular $\mathrm{T}$ cell formation and contributing to antibody maturation and selection.

Our case was in line with the predominant pattern reported in the literature, where most patients had presented MG manifestations prior to NMOSD. This could be caused by a deviation of immunity towards the predominant $\mathrm{T}$ helper 2 cell pathways, secondary to immunomodulatory therapy, thus increasing susceptibility to antibody-mediated disorders. The above is evidenced not only in patients with MG on pharmacological therapy and who subsequently presented with $\mathrm{NMO}^{7}{ }^{7}(7)$, but also in cases of NMO managed with low-dose interferon-beta $1 \mathrm{~b}$ or azathioprine who subsequently present MG. ${ }^{4,8}$

Consistent with the literature, our patient had a history of thymectomy prior to developing NMOSD. A rationale for this pattern, is based on the suppressor T cells contained in the thymus, which are responsible for inhibiting the spontaneous reaction of $\mathrm{B}$ cells, thus preventing autoimmune diseases. After thymectomy, patients have been shown to have increasing numbers in circulating CD4+ and CD8 $+\mathrm{T}$ cells, serum IgG and IgM, increased anti-DNA Abs, ANA Abs, anti-cardiolipin Abs and a decrease in regulatory $\mathrm{T}$ cells, with reports not only from NMOSD but also from other pathologies such as SLE. ${ }^{9-11}$

In addition, antibodies in NMO have been proposed to be a paraneoplastic response in MG patients with thymoma. ${ }^{7}$ Studies have proven that thymoma cells from patients with and without MG, express AQP4 in their cell membrane. Anti-AQP4 antibodies taken from the serum of patients with diagnosis of NMOSD bind to this site (12). In our case, there was no report from the Pathology Department available.

However, the coexistence of these two pathologies is evident in some cases where patients never underwent thymectomy. ${ }^{4-8,10,13,14}$ or presented NMO before having such surgical procedure done, ${ }^{4}$ suggesting that the thymus is not the only responsible factor.

Seropositive patients for both pathologies have an increased association (up to $38-75 \%$ ) with other autoantibodies, mainly, but not limited to, antiphospholipid, anticardiolipin, antinuclear (ANA), anti SSA and SSB, single and double stranded DNA antibodies, and rheumatoid factor. ${ }^{5}$ This association is evidenced in many reviewed reports. ${ }^{4,14-18}$ However, our patient showed negative results for the other immunological profile tests.

In most reports, cases of MG were mild or moderate, like our patient, with adequate response to immunomodulatory therapy. However, there are case reports of severe MG with fatal outcomes. One of these cases was associated with complications of $\mathrm{NMO}^{4}$ and another was associated with complications of SLE as the immunerelated pathology.18
There are reports of several patients with major neurological sequelae. In a multicenter study of 16 cases, 10 of them presented blindness or severe gait impairment, associated with the duration of disease and its late diagnosis. ${ }^{4}$. Our patient also presented with a major visual sequela at the time anti-AQP4 Abs were detected. This event had not been initially attributed to NMOSD, but upon diagnosis, immunomodulatory therapy was optimized, which is expected to decrease the relapse rate and long-term disability.

\section{Conclusion}

Publications in patients with coexistence of MG and NMOSD in Latin America are limited, which makes the description of this case meaningful, considering that its association is high and greater than expected by chance.

It is important for us to be familiar with its presentations, as well as with the increased risk of new autoimmune diseases. Suspected diagnosis, timely testing for antibodies and a complete immunological profile can change the treatment options and the long-term functional prognosis. There is a need for more reports in order to extend the statistical information and to improve the characterization of these patients, primarily in our Latin American region.

\section{Acknowledgments}

We acknowledge the NeuroNet group for their helpful academical review of the manuscript.

\section{Conflicts of interest}

The authors declare no conflict of interests.

\section{References}

1. Wang Z, Yan Y. Immunopathogenesis in myasthenia gravis and neuromyelitis optica. Front Immunol. 2017;8(DEC):1-14.

2. Romi F, Gilhus NE, Aarli JA. Myasthenia gravis: Clinical, immunological, and therapeutic advances. Acta Neurol Scand. 2005;111(2):134-141.

3. D.B. B, T.M. DB, E.M.L. O, et al. Demyelinating disease in patients with myasthenia gravis. Arq Neuropsiquiatr [Internet]. 2008;66(1):5-7.

4. Leite MI, Coutinho E, Lana-Peixoto M, et al. Myasthenia gravis and neuromyelitis optica spectrum disorder: A multicenter study of 16 patients. Neurology. 2012;78(20):1601-1607.

5. Shahmohammadi S, Doosti R, Shahmohammadi A, et al. Autoimmune diseases associated with Neuromyelitis Optica Spectrum Disorders: A literature review. Mult Scler Relat Disord [Internet]. 2019;27:350-363.

6. Yau GSK, Lee JWY, Chan TTK, et al. Neuromyelitis optica spectrum disorder in a chinese woman with ocular myasthenia gravis: First reported case in the chinese population. Neuro-Ophthalmology. 2014;38(3):140 144.

7. Balarabe SA, Adamu MD, Watila MM, et al. Neuromyelitis optica and myasthenia gravis in a young Nigerian girl. BMJ Case Rep. 2015;2015:10 13.

8. Etemadifar M, Abtahi S-H, Dehghani A, et al. Myasthenia Gravis during the Course of Neuromyelitis Optica. Case Rep Neurol [Internet]. 2011;3(3):268-273.

9. Uzawa A, Mori M, Iwai Y, et al. Association of anti-aquaporin-4 antibody-positive neuromyelitis optica with myasthenia gravis. J Neurol Sci [Internet]. 2009;287(1-2):105-107. 
10. Ikeguchi R, Shimizu Y, Suzuki S, et al. Japanese cases of neuromyelitis optica spectrum disorder associated with myasthenia gravis and a review of the literature. Clin Neurol Neurosurg [Internet]. 2014;125:217-221.

11. Gotkine M, Fellig Y, Abramsky O. Occurrence of CNS demyelinating disease in patients with myasthenia gravis. Neurology. 2006;67(5):881883.

12. Chan KH, Kwan JSC, Ho PWL, et al. Aquaporin-4 water channe expression by thymoma of patients with and without myasthenia gravis. $J$ Neuroimmunol. 2010;227(1-2):178-184.

13. Kay CSK, Scola RH, Lorenzoni PJ, et al. NMO-IgG positive neuromyelitis optica in a patient with myasthenia gravis but no thymectomy. J Neurol Sci [Internet]. 2008;275(1-2):148-50.

14. Jarius S, Paul F, Franciotta D, et al. Neuromyelitis optica spectrum disorders in patients with myasthenia gravis: Ten new aquaporin-4 antibody positive cases and a review of the literature. Mult Scler J. 2012;18(8):1135-1143
15. Furukawa Y, Yoshikawa H, Yachie A, et al.. Neuromyelitis optica associated with myasthenia gravis: characteristic phenotype in Japanese population. Eur J Neurol [Internet]. 2006;13(6):655-658.

16. Ikeda K, Araki Y, Iwasaki Y. Occurrence of CNS demyelinating disease in patients with myasthenia gravis. Neurology [Internet]. 2006;67(16):1326.

17. Tsujii T, Nishikawa N, Tanabe N, et al. Myasthenia gravis complicated with optic neuritis showing anti-aquaporin 4 antibody: a case report. Rinsho Shinkeigaku [Internet]. 2012;52(7):503-506.

18. Bibic VC, Brust TB, Burton JM. Neuromyelitis optica spectrum disorder presenting with concurrent autoimmune diseases. Mult Scler Relat Disord [Internet]. 2019;125-128. 